\title{
DHA-1 plasmid-mediated AmpC $\beta$-lactamase expression and regulation of Klebsiella pnuemoniae isolates
}

\author{
YING LUAN $^{1}$, GUI-LING LI ${ }^{1}$, LI-BO DUO ${ }^{1}$, WEI-PING WANG ${ }^{2}$, CHENG-YING WANG $^{2}$, \\ HE-GUANG ZHANG ${ }^{1}$, FEI HE ${ }^{1}$, XIN HE $^{1}$, SHU-JUAN CHEN ${ }^{3}$ and DAN-TING LUO ${ }^{4}$

\begin{abstract}
${ }^{1}$ Department of Medicine Laboratory, Second Affiliated Hospital of Harbin Medical University, Harbin, Heilongjiang 150086;
${ }^{2}$ Medicine Laboratory, Department of Urology Surgery, Daqing Oilfield General Hospital, Daqing, Heilongjiang 163001;

${ }^{3}$ Department of Medicine Laboratory, Hospital of Harbin Institute of Technology, Harbin, Heilongjiang 150001;

${ }^{4}$ Department of Medicine Laboratory, The Fourth People's Hospital of Shenyang, Shenyang, Liaoning 110031, P.R. China
\end{abstract}

Received October 25, 2013; Accepted July 9, 2014

DOI: $10.3892 / \mathrm{mmr} .2014 .3054$

\begin{abstract}
The present study aimed to investigate the regulatory mechanism of the $\mathrm{AmpC}$ enzyme by analyzing the construction and function of AmpCR, AmpE and AmpG genes in the Dhahran (DHA)-1 plasmid of Klebsiella pneumoniae (K. pneumoniae). The production of AmpC and extended-spectrum $\beta$-lactamase (ESBL) were determined following the cefoxitin (FOX) inducing test for AmpC, preliminary screening and confirmation tests for ESBL in 10 DHA-1 plasmid AmpC enzymes of $K$. pneumoniae strains. AmpCR, AmpD, AmpE and AmpG sequences were analyzed by polymerase chain reaction. The pACYC184-X plasmid analysis system was established and examined by regulating the pAmpC enzyme expression. The electrophoretic bands of AmpCR, AmpD, AmpE and AmpG were expressed. Numerous mutations in $\mathrm{AmpC}+\mathrm{AmpR}(\mathrm{AmpCR})$ and in the intergenic region cistron of AmpC-AmpR, AmpD, AmpE and AmpG were observed. The homology of AmpC and AmpR, in relation to the Morganella morganii strain, was $99 \%$, which was determined by comparing the gene sequences of Kp1 with those of Kp17 AmpCR. The specific combination of AmpR and labeled probe demonstrated a band retarded phenomenon and established a spatial model of AmpR. All the enzyme production strains demonstrated Val93 $\rightarrow$ Ala in AmpG; six transmembrane domains were found in AmpE in all strains, with the exception of $\mathrm{Kp} 1$ and $\mathrm{Kp} 4$, which had only three transmembrane segments that were caused by mutation. The DHA-1 plasmid AmpC enzymes encoded by plasmid are
\end{abstract}

Correspondence to: Dr Li-Bo Duo, Department of Medicine Laboratory, Second Affiliated Hospital of Harbin Medical University, No. 148 Baojian Road, Harbin, Heilongjiang 150086, P.R. China

E-mail: dlbdoccn@126.com

Key words: plasmid AmpC enzyme, Klebsiella pneumoniae, DHA-1, mutation, induction similar to the inducible chromosomal AmpC enzymes, which are also regulated by AmpD, AmpE, AmpR and AmpG.

\section{Introduction}

$\beta$-lactam is an effective antibiotic eliciting marginally toxic effects. This antibiotic has been widely used for a number of years. As such, certain bacteria produce hydrolases that induce resistance. Numerous factors contributing to antibiotic resistance have been determined; these factors include Capnocytophaga species, one of the most frequently identified organisms in the oral microbiome (1). A clinical survey has demonstrated that the overall prevalence of extended-spectrum $\beta$-lactamase (ESBL)-producing Klebsiella pneumoniae (K. pneumoniae) is $11.8 \%$ (2).

AmpC $\beta$-lactamase belongs to group I and class C according to the functional classification by Bush et al and the molecular classification by Ambler et al, respectively $(3,4)$. Due to high AmpC enzyme expression levels, bacteria are resistant to penicillin, cephalosporin generations I, II and III, cephamycins, monobactams and enzyme inhibitors (5); therefore, numerous studies have focused on this enzyme.

AmpC $\beta$-lactamases may be divided according to chromosome- and plasmid-mediated types. Although the mechanism of AmpC enzyme synthesis remains unclear, this enzyme is possibly regulated by an Amp composite operon, which is present in numerous Gram-negative bacteria containing AmpC as a structural gene, as well as AmpC, AmpR, AmpG, AmpD and $\mathrm{AmpE}$ as regulatory genes. The AmpC gene codes for the AmpC enzyme, which is then regulated by AmpR, AmpG, AmpD and AmpE (6-9). Although the AmpC enzyme may be translated from the AmpC gene, this enzyme has numerous mutations (10). AmpR and AmpC are arranged contiguously and are transcribed reversely to produce an AmpR protein; this protein is a negative regulatory factor of $\mathrm{AmpC}$ (11). With the emergence of $\beta$-lactamase antibiotics, AmpR activates AmpC to produce an $\mathrm{AmpC}$ enzyme; otherwise, $\mathrm{AmpR}$ functions as an AmpC suppressor $(6,11)$. AmpG is a transmembrane protein, a permease, which is able to transfer substances between the periplasmic space and the cytoplasm to AmpR by other proteins; therefore, AmpR may also function as an activator to 
promote AmpC (6). AmpD is translated from AmpD as a new type of $\mathrm{N}$-acetyl glucosamine-L-alanine amidase. AmpD is a negative regulator of $\beta$-lactamases and is located at the distal end next to the AmpE gene $(7,11,12)$. AmpE is situated at the downstream region of $\mathrm{AmpD}$, which is expressed coordinately with AmpD; however, the function of this gene in AmpC enzyme induction remains unclear (13).

Studies have demonstrated that strains expressing the AmpC enzyme contain high levels of AmpD, AmpR and AmpG; AmpD is a regulator of $A m p C$ by $A m p D$ protein, which negatively regulates the AmpC enzyme $(14,15)$. One cross-sectional study dealt with the isolation of bacteria from critically ill patients who had undergone tracheal intubation and mechanical ventilation; $32.9 \%$ of the bacterial strains were Acinetobacter spp. and $25.1 \%$ were $K$. pneumoniae, with Acinetobacter spp. the predominant AmpC $\beta$-lactamase producer (16). AmpC enzymes that are produced in plasmids also exhibit the same biochemical properties and resistance as chromosome types, as well as the same active sites identified by amino acid sequences analysis, including Ser-X-X-Lys at position 64 (the essential site of serine), Lys-Ser/Thr-Gly at position 315-317 (the essential site in active center tertiary structure) and tyrosine residue at position 150 (one important site of Thy-C-Asn in Class C enzyme that facilitates the hydrolysis of $\beta$-lactamases). With the induction of AmpC enzyme in the plasmid, certain cephalosporins cause resistance by inducing AmpC expression. The resistance is associated with AmpC, AmpD, AmpE, AmpR and AmpG, as chromosomal types; simultaneously, the resistance became stronger with the multiplication of the resistant strains that causes treatment failure clinically. The majority of the time, the resistance genes are transformed between the chromosome and plasmid by transposon, integron and insertion sequences (5); therefore, the resistance induced by plasmid-mediated AmpC enzyme causes more harm than that of chromosome-mediated AmpC.

Plasmid-mediated AmpC enzymes are commonly detected in K. pneumoniae (17). In a previous study, the levels of DHA-1 plasmid AmpC enzyme were detected locally; the sequences of AmpC + AmpR (AmpCR), AmpD, AmpE and AmpG genes in the DHA-1 plasmid of K. pneumoniae and the effect of the AmpD repressor gene on $\mathrm{pAmpC}$ were also identified. With the establishment of the pACYC184-X plasmid system, pAmpC regulation was revealed; meanwhile, AmpD mutation in the plasmid-mediated AmpC enzyme was detected by examining the sequence of the structural and promoter genes, as well as the whole gene. To understand the effect of AmpR on the AmpC enzyme, the transcriptional activity, space structure and AmpIR sequence of Kpl AmpR were detected, and the topological structure of AmpE and AmpG were simultaneously predicted. In conclusion, the resistance of strains that produce the AmpC enzyme was further understood, along with the development of a theory regarding the plasmid Amp operon.

\section{Materials and methods}

Strains and plasmid. Ten $K$. pneumoniae strains of DHA-1 plasmid (i.e., Kp1, Kp4, Kp11, Kp13, Kp17, Kp18, Kp19, $\mathrm{Kp} 20, \mathrm{Kp} 21$ and Kp22) were collected by the laboratory of the Second Affiliated Hospital of Harbin Medical University
(Harbin, China), but only Kp1 and Kp17 were selected for AmpD and AmpR regulation. The plasmids used were the following: K. pneumoniae-sensitive strains (Kps) that were collected from the laboratory of the Second Affiliated Hospital of Harbin Medical University, standard strains of Escherichia coli (E. coli) DH5a (Shanghai Shenyang BoCai Company, Shanghai, China), depressed sustained high-expressing AmpC Enterobacter cloacae (E. cloacae 029M; Zhejiang University, Hangzhou, Zhejiang, China), pGEM-T Easy vector plasmid (Promega Corporation, Madison, WI, USA), pACYC184 plasmid expression vector (product of NEB company, collected by Qingdao Marine Research Centre, Qingdao, Shangdong, China) and pKK232-8 promoter probe plasmid (Megiddo Bio-Pharm Technology Co., Ltd., Shanghai, China).

Antibiotic susceptibility of strains. All strains were analyzed separately using the disk diffusion method (Kirby-Bauer). Monoclonal colonies were mixed to form a solution with $0.5 \mathrm{MCF}$ concentration $\left(1.5 \times 10^{8} \mathrm{CFU} / \mathrm{ml}\right)$. The solution was placed on an $\mathrm{MH}$ agar plate using a sterile cotton swab, with cefoxitin (FOX) as an inducer in the center; piperacilin (PRL), ceftazidime (CAZ), cefotaxime (CTX), cefepime (CEP), cefoperazone/sulbactam (SCF), imipenem (IPM), piperacillin/ tazobactam (TZP) and aztreonam (ATM) were placed at a distance of 20-22 mm from the center of the FOX disk. Following this, the $\mathrm{MH}$ agar plates were incubated at $35^{\circ} \mathrm{C}$ for 18-20 h to observe the bacterial inhibition zone diameters.

Phenotype examination of resistant strains. Complex phenotype analysis was designed in reference to Moland et al $(18,19)$. ESBL and AmpC induction may be observed on the same plate simultaneously. FOX and AMC were induced at the center, whereas PRL, CAZ, cefepim (FEP), CTX, ATM, TZP and ceftriaxone (CRO) surrounded the periphery and were distributed evenly. The plate was cultured at $35^{\circ} \mathrm{C}$ for $18-20$ h. E. coli ATCC25922 was selected as the negative control strain and E. cloacae 029 was selected as the inducible AmpC enzyme positive control strain. The results were categorized according to the following criteria: ESBL-positive (the bacteriostasis circle close to the AMC side expanded or widened), inducible AmpC enzymes (the bacteriostasis circle around FOX or close to FOX became flat or depressed) and sustainable yield AmpC enzyme (the edge of the bacteriostasis circle became smooth).

Amplification of chromosomal DNA and preparation of the plasmid template. The stains were inoculated on blood agar following isolation and were cultured overnight at $37^{\circ} \mathrm{C}$. A single colony was selected to inoculate the $5 \mathrm{ml} \mathrm{LB}$ liquid medium of FOX (with a final concentration of $16 \mu \mathrm{g} / \mathrm{ml}$ ), then incubated in a shaking water bath at $200 \mathrm{r} / \mathrm{min}$ and $37^{\circ} \mathrm{C}$ for 18-20 h. Subsequently, the chromosomal DNA was extracted from $3 \mathrm{ml}$ culture medium, and the plasmid DNA was extracted from 1-2 $\mathrm{ml}$ culture medium. The procedure was performed according to the manufacturer's instructions of the E.Z.N.A. ${ }^{\circledR}$ bacterial DNA kit and the E.Z.N.A. plasmid mini kit I (Omega Bio-Tek Inc., Norcross, GA, USA), respectively.

Sequence analysis of the AmpC enzyme gene. For the AmpCR, AmpD, AmpE and AmpG genes, the specific primers were designed in the DHA-1 plasmid AmpC enzyme K.pneumoniae, 


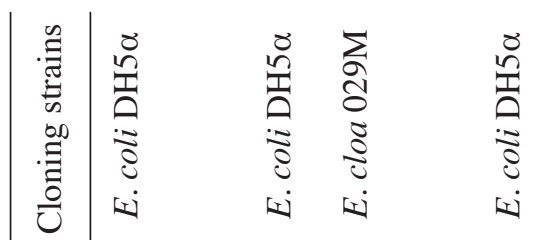

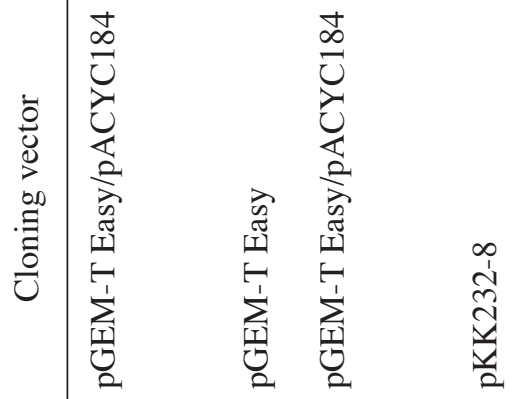

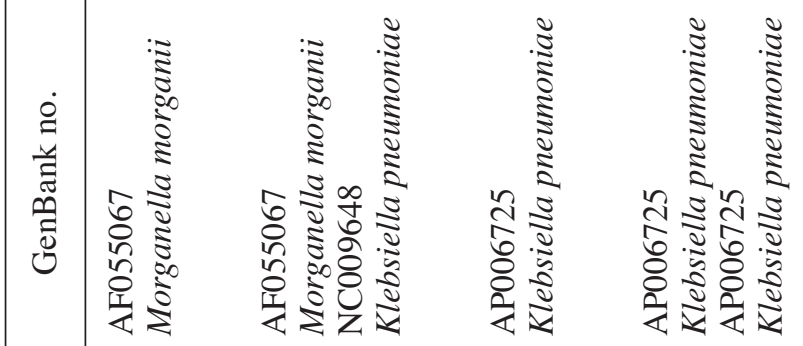

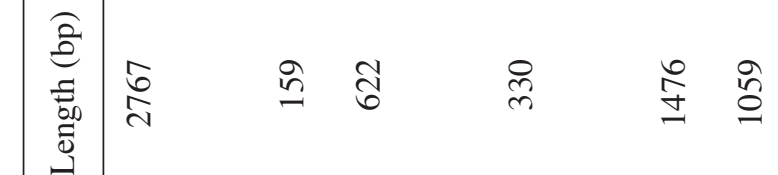

密

告

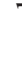

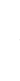

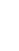

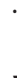

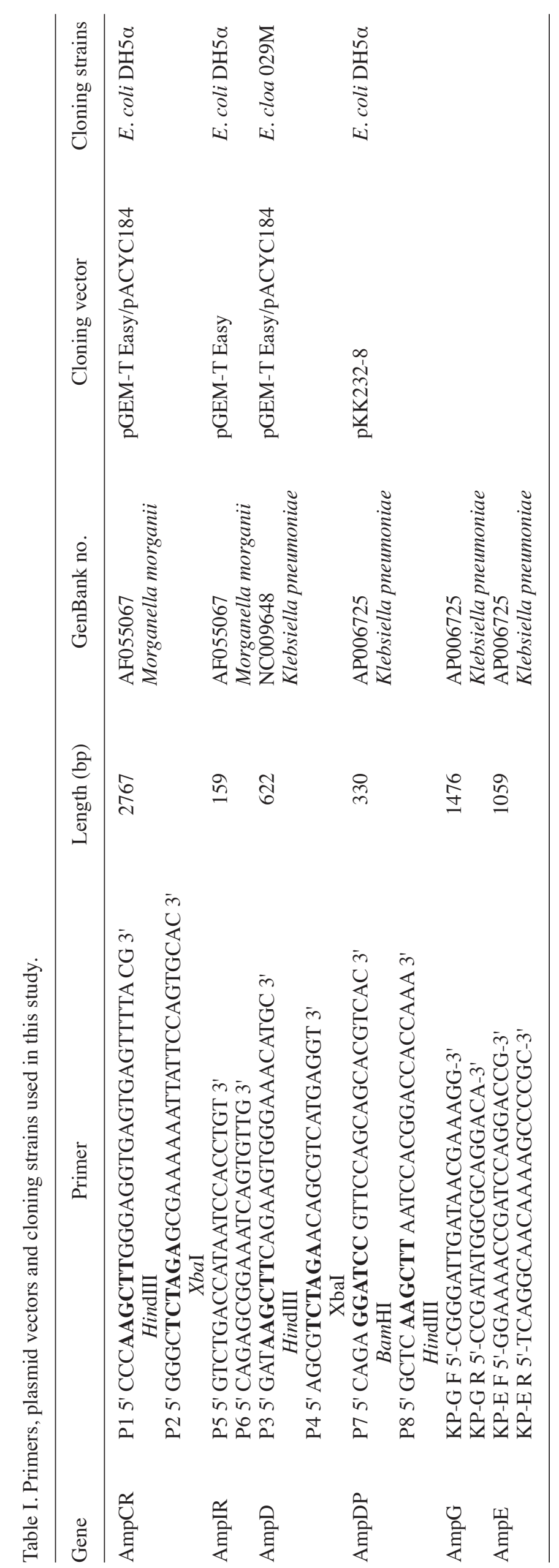


according to the Morganella morganii (M.morganii) strain on GenBank bacteria (AmpCR), K. pneumoniae MTUH-K2044 (AmpD) and K. pneumoniae AP006725 (AmpE and AmpG) (Table I). The plasmid AmpCR gene and chromosomal AmpD, AmpE and AmpG genes were amplified and purified by polymerase chain reaction (PCR) using primers P1-P2, P3-P4, KPGF-KPGR and KPEF-KPER, shown in Table I. AmpCR and AmpD PCR amplification were performed for 30 cycles of $10 \mathrm{sec}$ each denaturation at $98^{\circ} \mathrm{C}$, annealing at $56^{\circ} \mathrm{C}$ for $5 \mathrm{sec}$, extension at $72^{\circ} \mathrm{C}$ for $2 \mathrm{~min}$ and $30 \mathrm{sec}$ and a final extension at $72^{\circ} \mathrm{C}$ for $7 \mathrm{~min}$. AmpG PCR amplification was performed for initial denaturation at $94^{\circ} \mathrm{C}$ for $5 \mathrm{~min}$, 35 cycles of $30 \mathrm{sec}$ each denaturation at $94^{\circ} \mathrm{C}$, annealing at $51^{\circ} \mathrm{C}$ for $30 \mathrm{sec}$, extension at $72^{\circ} \mathrm{C}$ for $30 \mathrm{sec}$ and a final extension at $72^{\circ} \mathrm{C}$ for $7 \mathrm{~min}$. AmpE PCR amplification was performed for initial denaturation at $95^{\circ} \mathrm{C}$ for $4 \mathrm{~min}, 30$ cycles of $30 \mathrm{sec}$ each denaturation at $95^{\circ} \mathrm{C}$, annealing at $58^{\circ} \mathrm{C}$ for $30 \mathrm{sec}$, extension at $72^{\circ} \mathrm{C}$ for $30 \mathrm{sec}$ and a final extension at $72^{\circ} \mathrm{C}$ for $7 \mathrm{~min}$. The products of AmpCR (AmpC $+\mathrm{AmpR}+\mathrm{AmpIR})$ and AmpD were conjugated to the pGEM-T Easy carrier and transformed into $E$. coli DH5 $\alpha$-competent cells. The recombinant colonies were screened by a blue-white plaque selection to examine the sequences of the recombinants and compare these colonies with those of the M. morganii strain. The sequences of the AmpE and AmpG genes were compared with those of the AmpE and AmpG genes in the antibiotic-sensitive Kp (Kps), and to detect AmpCR, AmpD, AmpE and AmpG genes in the DHA-1 plasmid AmpC enzymes. All sequence analysis was conducted by Beijing Sunbiotech Co., Ltd. (Beijing, China), and the genes were all compared with the sequences in GenBank by Blast (http://blast.ncbi.nlm.nih.gov/Blast.cgi).

The chromosomal AmpD structural gene was amplified by PCR and conjugated to the pGEM-T Easy carrier to form pT-AmpD, whose sequence was also detected. The site of the AmpD gene promoter was predicted by Softberry Fgenesh (http://www.softberry.com) for sequence analysis. The AmpDP PCR procedure was performed with initial denaturation at $94^{\circ} \mathrm{C}$ for $5 \mathrm{~min}, 30$ cycles of $30 \mathrm{sec}$ each denaturation at $94^{\circ} \mathrm{C}$, annealing at $62^{\circ} \mathrm{C}$ for $30 \mathrm{sec}$, extension at $72^{\circ} \mathrm{C}$ for $45 \mathrm{sec}$ and a final extension at $72^{\circ} \mathrm{C}$ for $5 \mathrm{~min}$. A recombinant carrier, pK-AmpDP, was identified by the conjugation of the promoter sequence to $\mathrm{pKK} 232-8$ promoter probe plasmid, and transformed into $E$. coli $\mathrm{DH} 5 \alpha$, which was selected from the double-resistant plates of ampicillin and chloramphenicol.

Effect of exogenous AmpCR and AmpD on AmpC enzyme induction of host bacteria. The AmpCR gene was cloned into pACYC184 carrier in Kp1 and Kp17 strains to form the recombinant plasmid pACYC184-AmpCR. The plasmid was then transformed into $E$. coli $\mathrm{DH} 5 \alpha\left(\mathrm{AmpCR}^{-} / \mathrm{AmpD}^{+}\right)$to evaluate the AmpC enzyme expression in recombinant E-AmpCR with the exogenous AmpCR induced into the $\mathrm{AmpCR}^{-}$ $\mathrm{AmpD}^{+}$strain. The AmpD gene was cloned into the pACYC184 carrier to form the recombinant plasmid pACYC184-AmpD. The plasmid was then transformed into E. cloacae $029 \mathrm{M}$ (AmpCR ${ }^{+} / \mathrm{AmpD}^{-}$) with the a high level of AmpC expression sustained, due to the AmpD mutation to examine the AmpC enzyme expression in recombinant Ea-AmpD with the exogenous $\mathrm{AmpD}^{+}$induced into the $\mathrm{AmpCR}^{+} / \mathrm{AmpD}^{-}$strain. The impact on AmpC enzyme in the host strain by recombinant plasmid pACYC184-AmpCR and pACYC184-AmpD was evaluated by examination of the induced resistance phenotype and the FOX minimum inhibitory concentration (MIC). All primers, cloning carriers and cloning strains are listed in Table I.

Electromobility shift assay (EMSA) of AmpIR and AmpR. The interaction between AmpIR and AmpR was detected using EMSA with the mark of biotin probes and chemiluminescent detection technology in Kp1 (DHA-1 plasmid AmpC enzyme K. pneumoniae) and purified recombinant AmpR. The AmpC-R (including AmpR, AmpIR and AmpC) was amplified using PCR using primers P1 and P2, shown in Table I, and conjugated with the pGEM-T Easy carrier, prior to sequencing using an ABI Prism3100 automated DNA sequencer (Applied Biosystems Life Technologies, Foster City, CA, USA). The nucleotide and amino acid sequences were analyzed using the BLAST program of the NCBI. The 3D structure of AmpR was established according to the sequence of amino acids, with Pseudomonas aeruginosa ( $P$. aeruginosa) transcriptional regulator PAO477 as the template by Swiss-Model server (http://www.swissmodel.expasy.org) and SPdviewer 4.0.1 (http://www.swissmodel.expasy.org).

Topology prediction analysis of AmpE and AmpG. The transmembrane domains of all DHA-1 K. pneumoniae and Kps were predicted by SOSUI engine ver. 1.1 (http://bp.nuap.nagoya-u. ac.jp/sosui/sosui_submit.html). The AmpE and AmpG transmembrane domains in the experiment and sensitive strains were also analyzed, as well as the functions of these enzymes.

Ethical approval. The study was approved by the ethics committee of the Second Affiliated Hospital of Harbin Medical University.

\section{Results}

Analysis of Amp protein expression and gene mutation. All the electrophoretic bands were at their expected positions following amplification by PCR with the specific primers.

Mutations and amino acid changes were analyzed by comparing them with the corresponding sequences of M. morganii (Genbank No.AF055067) and K. pneumoniae strains that were sensitive to all the antibiotics tested, using Align of the BLAST program. All ten revealed the same AmpR mutations (Thr114 $\rightarrow$ Ala). AmpG mutations were identified in all K. pneumoniae strains compared with the strains that were sensitive to all the antibiotics. The changes in the amino acids were: i) Silent mutations: Kp20, Kp21 and Kp22; ii) site 97 alanine mutated to threonine (Ala97 $\rightarrow$ Thr): Kp1, Kp4; iii) isoleucine transformed into leucine (Ile19 $\rightarrow$ Leu); iv) site 97 alanine mutated to threonine (Ala97 $\rightarrow$ Thr): Kp11, Kp13, Kp17, Kp18 and Kp19 (Table II).

Sequence alignment and location of AmpD promoter. The DNA fragment, amplified by PCR of the AmpD promoter with a length of $\sim 330 \mathrm{bp}$, was linked to the pKK232-8 probe plasmid with the CAT gene and was transfected into $E$. coli $\mathrm{DH} 5 \alpha$-competent cells. The -10 box of the AmpD promoter in K. pneumoniae was located upstream, at the -19 to -24 position, and the sequence 
Table III. Cefoxitin MIC in recombinant and strains.

\begin{tabular}{llcc}
\hline Strain & \multicolumn{1}{c}{ Plasmid features } & Cefoxitin MIC $(\mu \mathrm{g} / \mathrm{ml})$ & Resistance phenotype \\
\hline E. coli $\mathrm{DH} 5 \alpha$ & AmpCR $^{-}, \mathrm{AmpD}^{+}$ & $<16$ & None \\
E. cloacae $029 \mathrm{M}$ & AmpCR $^{+}$, AmpD $^{-}$ & $>1024$ & Over expression \\
Kp1 & AmpCR $^{+}$, AmpD $^{+}$ & 256 & Induced \\
Kp17 & AmpCR $^{+}$, AmpD $^{+}$ & 512 & Induced \\
E-pACYC184 & pACYC184 & $<16$ & None \\
E-AmpCR1 & pACYC184-AmpCR1 & 128 & Induced \\
E-AmpCR17 & pACYC184-AmpCR17 & Induced \\
Ea-AmpD1 & pACYC184-AmpD1 128 & Induced \\
Ea-AmpD17 & pAC YC184-AmpD17 & 256 & Induced
\end{tabular}

MICs, minimum inhibitory concentration. E. coli, Escherichia coli; E. cloacae, Enterobacter cloacae.

Table IV. Prediction of the AmpG transmembrane regions of Klebsiella pneumoniae encoding DHA-1-type plasmid-mediated AmpC $\beta$-lactamase.

\begin{tabular}{cclcc}
\hline Transmembrane domain & N-terminal & Sequence of transmembrane domain & C-terminal & Length (AA) \\
\hline 1 & 11 & QPKSAILLILGFASGLPLALTSG & 33 & 70 \\
2 & 48 & TIGFFSLVGQAYVFKFLWSPLMD & 104 & 23 \\
3 & 82 & GWLLTTQVLLLLAIAAMGFLEPV & 166 & 196 \\
4 & 144 & GAAISVLGYRLGMLVSGGLALWL & 247 & 33 \\
5 & 174 & QGMYWLMAALLVPCIIATLLAPE & 23 \\
6 & 225 & WLILLLIVLYKLGDAFAMSLTTT & 343 \\
7 & 278 & YGGVLMQRLTLFRALLIFGLLQG & 374 \\
8 & 321 & TAVFFENLCGGMGTAAFVALLMT & 405 \\
10 & 352 & TQFALLSALSAVGRVYVGPIAGW & 23 \\
12 & 383 & TFYLFSVVAAVPGIALLLLCRQT & 23 \\
\hline
\end{tabular}

Table V. Prediction of the AmpE transmembrane regions of Klebsiella pneumoniae encoding DHA-1-type plasmid-mediated AmpC $\beta$-lactamase.

\begin{tabular}{lrlrr}
\hline Transmembrane domain & N-terminal & Sequence of transmembrane domain & C-terminal & Length (AA) \\
\hline 1 & 1 & MTLFTTLLVLIAERLFKLGEHWQ & 23 & 23 \\
2 & 38 & FSLPGTLLMTLVAVAVVYIIQRL & 60 & 23 \\
3 & 65 & LFNIPLLVFWILLGLLCIGAGK & 86 & 171 \\
4 & 149 & FWFVVGGAWGPLTLIAYSVLRAW & 211 & 23 \\
5 & 189 & IDGILHIVDWLPVRLVGVVYALV & 284 \\
6 & 266 & TSLVVVVIMALLTIYGTLV & 19 \\
\hline
\end{tabular}

AA, amino acid.

was TGAGTT (as predicted by Softberry Fgenesh gene finder). Additionally, the -35 box was located upstream, at approximately -53 to -58 , and the sequence was TTCATC. No nucleotide mutation was found in the internal sequence between the structural and promoter genes of AmpD K. pneumoniae compared with the FOX-susceptible strains, whereas the mutation at the structural and promoter genes were the same.

FOX-resistant phenotype and MIC of recombinant E-ampCRI and E-ampCR17. Host strain E. coli DH5a was initially 


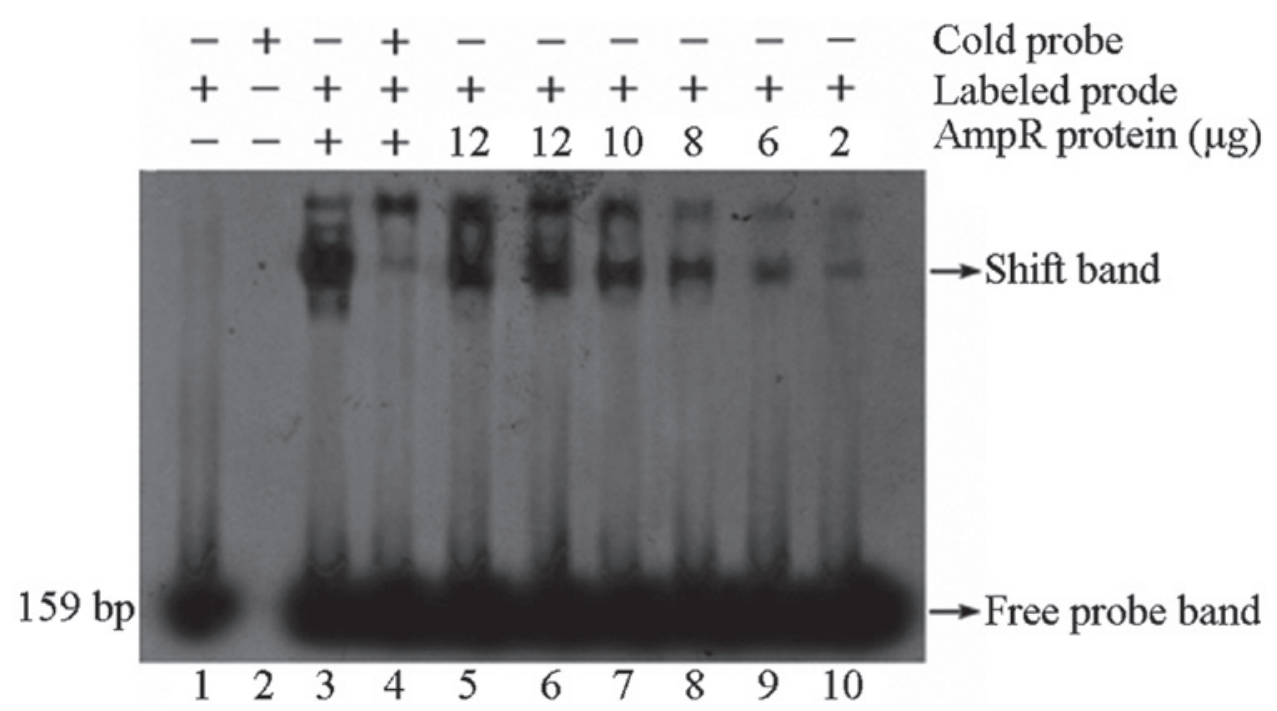

Figure 1. Retarded band of AmpIR probe and AmpR recombinant protein. The 159-bp AmpIR probe joined with the AmpR recombinant protein to form the retardation band, which was inhibited by the cold probe (with a concentration 100-fold that of the labeled probe). Lane 1, labeled probe; lane 2, unlabeled probe; lane 3, labeled probe + AmpR protein; lane 4, unlabeled probe + labeled probe + AmpR protein; lane 5-10, labeled probe + AmpR protein $(12,12,10,8,6$ and $2 \mu \mathrm{g})$.

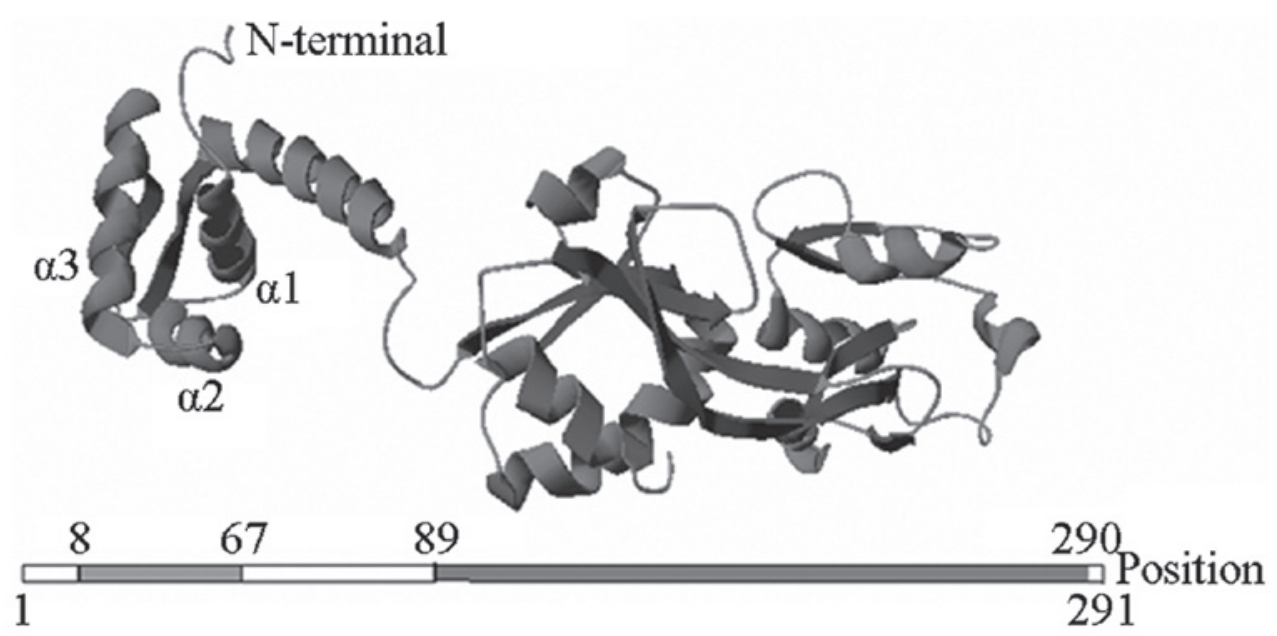

Figure 2. Simulated three-dimensional structure of AmpR regulatory protein. The helix-turn-helix domain of the amino-terminal, LEU8-GLU67, is evident and contains three $\alpha$-helices $(\alpha 1, \alpha 2$ and $\alpha 3)$. The auxiliary inducer binding domain position is 89His-290Ala.

sensitive to all of the antibiotics, and marked resistance was induced by the transfection of exogenous AmpCR. E-AmpCR1 and E-AmpCR17 (pAC YC184-AmpCR) recombinants exhibited inducible resistance to all tested antibiotics, wherein the bacterial inhibition zone was flattened with the FOX or IPM disk as an inducer in the plate center. The MIC value of FOX increased by eight-fold in E-AmpCR1 and E-AmpCR17 compared with the host strain E. coli DH5 $\alpha$, from 16 to $128 \mu \mathrm{g} / \mathrm{ml}$, and the MIC value demonstrated no change when the free conversion of E-pACYC184 vector plasmid was the recombinant. Therefore, the susceptibility of the host strain was not affected by the pACYC184 vector (Table III).

Comparison of repressor gene sequences in AmpD-induced strains by PCR and the enzyme digestion of cloning vectors. The nucleotide and amino acid homology of the AmpD gene with Kp1 and Kp sensitivity were both $100 \%$; while the nucleotide and amino acid homology with Kp17 were both
99\%; the 143 leucine changed to isoleucine ( $\mathrm{Leu} \rightarrow \mathrm{Ile}$; data not shown).

Structure and resistant phenotype of the pACYC184-AmpD plasmid vector. The AmpD of $\mathrm{Kp} 1$ and $\mathrm{Kp} 17$ was linked to XbaI (at position 1425) and HindIII (at position 1524), and the restriction sites of the pACYC184 plasmid vector, respectively; therefore, the plasmid that carried AmpD became the repressor of AmpC $\beta$-lactamase. The Ea-AmpD1 and Ea-AmpD17 (pAC YC184-AmpD) recombinants revealed an inducible resistance to CAZ when FOX or IPM were present as inducers. The MIC of FOX on Ea-AmpD1 and Ea-AmpD17 decreased to $256 \mu \mathrm{g} /$ $\mathrm{ml}$, whereas the MIC of the E. cloacae $029 \mathrm{M}$ host strain was $>1,024 \mu \mathrm{g} / \mathrm{ml}$. Therefore, the AmpD gene, from K. pneumoniae, has a function in partial compensation (Table III).

Alignment analysis of the AmpIR gene and structural modeling of the AmpR protein. The DNA-protein interaction system, 
which was established by an AmpIR probe (biotin-labeled and unlabeled competing probe) and recombinant protein AmpR of K.pneumoniae, demonstrated that the AmpR protein would bind to the probe specifically, and a significant retardation phenomenon would be observed. The suppression was more evident with the increase in protein concentration (Fig. 1). The AmpCR sequence was $2186 \mathrm{bp}$, amplified with primers P1 and $\mathrm{P} 2$, and the GenBank number was HM568877.1. This sequence contains AmpR, AmpC and AmpIR genes, which are the intercistronic sequences of the AmpC and AmpR genes. Using Blast in NCBI, the homology of AmpIR and the M. morganii strain (GenBank no. AF055067) was identified to be 97\%, with three base mutations: $915 \mathrm{~T} \rightarrow \mathrm{A}, 933 \mathrm{~T} \rightarrow \mathrm{C}$ and $961 \mathrm{C} \rightarrow \mathrm{T}$. Similarity was observed between the DNA biting site of the 38-bp AmpR protein in the Kpl plasmid and the AmpIR, with a T-N11-A sequence and a unique palindromic region. AmpR protein, which comprises 291 amino acids (EU476911.1), demonstrated one amino acid mutation $(114 \mathrm{~T} \rightarrow \mathrm{A})$ when compared with the M. morganii strain (AF055067). The structure of AmpR protein was modeled with $P$. aeruginosa $\mathrm{PAO} 477$ regulatory protein (PDB: 2esnB) as the homology template, and was modified by Swiss-Model server and associated software. A helix-turn-helix domain was identified at the AmpR amino terminus, and an auxiliary inducer binding domain was found at the carboxyl terminus (Fig. 2).

Prediction of transmembrane domain of AmpG in DHA-1 plasmid AmpC enzyme K. pneumoniae. Predictions were performed using Expasy online software SOSUI engine Twelve transmembrane domains were identified in the protein. The N-terminus and $\mathrm{C}$-terminus were exposed outside of the membrane. The transmembrane domains were located at positions 11-33, 48-70, 82-104, 144-166, 174-196, 225-247, 278-300, 321-343, 352-374, 383-405, 426-448 and 461-483 (Table IV). The Ile19 $\rightarrow$ Leu, Ala $97 \rightarrow$ Thr, Val441 $\rightarrow$ Ala and Ala444 $\rightarrow$ Gly mutations were found in the transmembrane domains (Table IV).

Prediction of transmembrane domain of AmpE in DHA-1 plasmid AmpC enzyme K. pneumoniae. The transmembrane domains of AmpE were predicted by SOSUI and six transmembrane domains in the strains were found. However, Kp1 and Kp4 had a frameshift mutation (155) caused by a base deletion $(465 \Delta \mathrm{C})$ and, therefore, only three transmembrane domains were identified (Table V).

\section{Discussion}

$K$. pneumoniae is one of the most important pathogens that causes clinical infections and nosocomial infections. The wild type of $K$. pneumoniae is resistant to ampicillin. With the abuse of antibiotics, such as cephalosporins III, bacteria produce ESBLs, which may be suppressed by $\beta$-lactamase inhibitors, such as clavulanic acid (20). In the present study, the gene and amino acid mutations of AmpCR, AmpD, AmpE and AmpG of the DHA-1 plasmid AmpC enzyme were observed in ten $K$. pneumoniae strains. A high homology was found in the base sequence of AmpCR between the plasmid and chromosome types; and the conserved region of AmpR and its cistron fragment was notably longer than that for AmpC. The interval region of the cistron in the plasmid contained AmpR and AmpC promoters, and a number of the base pairs belonged to both of the promoters, which coincides with the regulation of the AmpC enzyme chromosome $(21,22)$ and plasmids (23). High homology was noted between the interval region of the cistron in the AmpC of the Kp strain and chromosome of M. morganii bacteria, where DNA binding sites of AmpR protein that contains T-N11-A sequence and palindrome structure are found. AmpR belongs to LysR regulatory protein family, which is one of the transcription factors of AmpC enzymes (24) that may inhibit AmpC synthesis when $\beta$-lactamase enzyme is absent, and promote AmpC synthesis, with $\beta$-lactam antibiotics as the inducer. To gain structural insights into AmpR regulation, a crystal structure of the effector binding domain (EBD) was determined; the base of the interdomain pocket of this crystal structure inhibited AmpR from inducing AmpC (Thr103Val, Ser221Ala and Tyr264Phe) or resulted in constitutive AmpC expression (Gly102Glu) (25). The resistance gene may be transfected by transposons, integrons and insertion sequences, for example, from a chromosome to the plasmid or a plasmid to a plasmid. For example, in producing a plasmid-mediated CFE-1 type AmpC enzyme in E. coli KU640, an insertion sequence 26 (IS26) was found in addition to AmpR and AmpC, which was able to transfer the Amp gene (26). From the FOX-resistant $K$. pneumoniae strains, the majority of AmpC genes were Miriam or DHA type, which transfer the resistance carried by the plasmid to the recipient strain by conjugation (27).

The resistance to drugs that is spread between bacterial species by plasmids was the most important mechanism, and marginal differences were noted on the sites and functions of the genes, either encoded by plasmid or chromosome; however, the observations remained different among species (28). For example, the DHA-2-type AmpC enzyme plasmid-mediated $K$. pneumoniae is the point-mutant strain of the DHA-1 type AmpC enzyme gene, and has a 99\% homology with M. morganii (29). In the present study, EMSA demonstrated that AmpR binding with AmpIR is the transcription factor of the plasmid AmpC enzyme. The structure of the AmpR protein is similar to the LysR family with a helix-turn-helix domain at the $\mathrm{N}$ terminus, which was also constructed in the three-dimensional model of AmpR.

No deletion mutations of the regulatory gene were observed among the 10 strains in the present study, including AmpD, AmpE and AmpG. No AmpD or AmpG mutation was observed in some strains that were used in the present study; however, all of the strains with AmpE mutation were compared with the FOX-susceptible strains. Although mutation occurred in the strains, no strain was identified with the phenotype and MIC of FOX.

AmpD is a cytoplasmic peptidoglycan amidase that is involved in baterial cell-wall recycling and is a key enzyme in $\beta$-lactamase induction (30). AmpD represses the inducible expression of the AmpC enzyme. Mutations in the promoter or the structural gene of AmpD are able to abrogate the repression of AmpC enzyme transcription, and inactivation of AmpD significantly increases AmpC expression. The role of AmpD in entry into the cell wall, recycling events and in reversal of the induction production of $\beta$-lactamase has been proposed as a possible factor in the development of antibiotic 
resistance (31). At present, the chromosomal AmpD gene was found in K. pneumonia strains. The effect of AmpD on the inducible expression in the presence of DHA-1 type AmpC enzyme with AmpR was examined with the pACYC184-X plasmid evaluation system. Transformation resulted in a decrease in cefoxitin MICs and an inducible phenotype of $\beta$-lactams. Thus, the AmpD gene of the Kp1 strain may complement the AmpD mutant background of E. cloacae 029M, in part. These findings highlight the importance of AmpD in the regulation of the K. pneumonia AmpC cephalosporinase production. The location of the AmpD promoter region remains unknown. The present study identified putative promoter -10 box and -35 box regions located $-19--24$ bp and -53 - 58 bp upstream of AmpD using Softberry FGENESH online, by constructing the recombinant carrier $\mathrm{pK}-\mathrm{AmpDP}$ which contained the upstream nucleotide sequence of AmpD.

Currently, the majority of studies regarding AmpE focus on the polymorphism, topology prediction and polar characteristics, but not on the function of the gene. AmpE is an intracellular membrane protein composed of 284 amino acids, with a molecular weight of $31.2 \mathrm{kDa}$. The transmembrane domain suggests that AmpE is a receptor on the membrane, such as anchored protein or ion channel protein (32). In 1989, Lindquist et al (33) found that AmpE was adjacent to AmpD in the chromosome of E. coli $\mathrm{K} 12$, forming the AmpDE composite operon, and AmpR, AmpR-AmpC, AmpD, AmpE, and AmpD-AmpE cloning plasmids were constructed with E. coli JRG582 $\Delta\left(\mathrm{AmpD}^{+} / \mathrm{AmpE}^{+}\right) 2$; the cloned plasmids were then transformed into E. coli JRG582, an AmpDE deletion strain, to find the expression and induction of $\beta$-lactamase based on the Amp compound operon with the deletion of different regulation genes. The results demonstrated that induced $\beta$-lactam expression in AmpC enzyme was not affected by AmpE in E. coli JRG582 (AmpR ${ }^{+} / \mathrm{AmpC}^{+} / \mathrm{AmpD}^{+}$ /AmpE-), i.e., AmpD was suppressed independently; in E. coli JRG582 (AmpR $\left.{ }^{+} / \mathrm{AmpC}^{+} / \mathrm{AmpD}^{-} / \mathrm{AmpE}^{+}\right)$, the endogenic $\beta$-lactamase expression was effected by AmpE, which means AmpE enhanced the repression effect of AmpD. The results overturned the hypothesis of Honoré et al (34), who proposed that AmpE is the signal transduction protein in the process of AmpC enzyme induction, and the induction of AmpC is completely disrupted without AmpE although AmpR is present. Only three transmembrane segments of AmpE are present in $\mathrm{Kp} 1$ and $\mathrm{Kp} 4$, but no changes in induction and resistance to FOX are observed. Therefore, AmpC enzyme would not be induced by AmpE independently with the expression of AmpD, AmpG and AmpCR, and no effect on AmpC enzyme was observed with the AmpE mutation. With the cloned normal and mutation sequences of AmpE on the chromosome of K. pneumoniae, AmpE was predicted as a transmembrane protein, which may be used in further investigations on the regulation and molecular biological characteristics of AmpC.

The AmpG gene encodes a transmembrane protein (permease) that transports peptide murein debris from the periplasm to the cytoplasm, which serve as signal molecules for the induction of AmpC $\beta$-lactamase (35). In the present study, the MIC values was extremely high in four strains, with two point mutations (Ile19 $\rightarrow$ Leu and Ala97 $\rightarrow$ Thr) in five strains, but had little association with the AmpG mutation.
The structure of AmpG in K. pneumoniae, as predicted by the SOSUI system, was different from the AmpG in E. coli predicted by Chahboune et al (36). A total of 12 transmembrane segments and a large cytoplasmic loop B, with $\mathrm{N}$-terminus and $\mathrm{C}$-terminus outside the cytomembrane were found; whereas, 10 transmembrane segments and two large cytoplasmic loops of the AmpG were predicted in E. coli. Schmidt et al (37) obtained three mutant strains of AmpG by nitrosoguanidine E. coli SN0301 (with E. cloacae AmpCR), which were Gly151 $\rightarrow$ Asp151, Gly268 $\rightarrow$ Asp268 and Gly373 $\rightarrow$ Asp373, and all the mutations led to the loss of inducibility and could be recovered with AmpG recovery. AmpG stability was affected by Gly268 $\rightarrow$ Asp268, which was at the beginning of a transmembrane segment; and the inducing signal transduction was affected by Gly151 $\rightarrow$ Asp151 and Gly373 $\rightarrow$ Asp373, which were located at the two large cytoplasmic loop. The amino acid mutations found in the present study (Ile19 $\rightarrow$ Leu, Ala $97 \rightarrow$ Thr) were in the inside surface of the transmembrane domains, which had no effect on the function of AmpG. Mutations of AmpG, affecting the inducible AmpC $\beta$-lactamase production, have been demonstrated, through the use of chemical mutagenesis methods described in previous studies $(37,38)$. However, in the present study, spontaneous mutations of the AmpG gene in K. pneumoniae isolates did not have a significant effect on the induction of AmpC. Further investigations are thus required in order to clarify the role of the regulatory genes, AmpR, AmpD, AmpE and AmpG, and their corresponding gene products, in the induction of plasmid-mediated AmpC $\beta$-lactamase expression. Additional areas of future research include the role of penicillin-binding proteins or transmembrane proteins as sensors for induction, as well as the interaction of AmpC, AmpR, AmpD, AmpE and AmpG. With the association between all mutations of the AmpG and AmpC enzymes induced, the resistance to $\beta$-lactam antibiotics may be partially overcome.

\section{Acknowledgements}

This study was supported by the Natural Science Foundation of Heilongjiang Province of China (grant no. H2013100).

\section{References}

1. Ehrmann E, Handal T, Tamanai-Shacoori Z, Bonnaure-Mallet M and Fosse T: High prevalence of $\beta$-lactam and macrolide resistance genes in human oral Capnocytophaga species. J Antimicrob Chemother 69: 381-384, 2014.

2. Raji MA, Jamal W, Ojemhen O and Rotimi VO: Point-surveillance of antibiotic resistance in Enterobacteriaceae isolates from patients in a Lagos Teaching Hospital, Nigeria. J Infect Public Health 6: 431-437, 2013.

3. Bush K, Jacoby GA and Medeiros AA: A functional classification scheme for beta-lactamases and its correlation with molecular structure. Antimicrob Agents Chemother 39: 1211-1233, 1995.

4. Ambler RP, Coulson AF, Frère JM, et al: A standard numbering scheme for the class A beta-lactamases. Biochem J 15: 269-270, 1991.

5. Philippon A, Arlet G and Jacoby GA: Plasmid-determined AmpC-type beta-lactamases. Antimicrob Agents Chemother 46: 1-11, 2002.

6. Garcia DL and Dillard JP: Mutations in ampG or ampD affect peptidoglycan fragment release from Neisseria gonorrhoeae. J Bacteriol 190: 3799-3807, 2008.

7. Jacobs C: Pharmacia Biotech \& Science prize. 1997 grand prize winner. Life in the balance: cell walls and antibiotic resistance. Science 278: 1731-1732, 1997. 
8. Wiedemann B, Pfeifle D, Wiegand I and Janas E: beta-Lactamase induction and cell wall recycling in gram-negative bacteria. Drug Resist Updat 1: 223-226, 1998.

9. Jacobs C, Joris B, Jamin M, et al: AmpD, essential for both $\beta$-lactamase regulation and cell wall recycling, is a novel cytosolic $\mathrm{N}$-acetylmuramyl-L-alanine amidase. Mol Microbiol 15: 553-559, 1995.

10. Yu WL, Ko WC, Cheng KC, et al: Institutional spread of clonally related Serratia marcescens isolates with a novel AmpC cephalosporinase (S4): a 4-year experience in Taiwan. Diagn Microbiol Infect Dis 61: 460-467, 2008.

11. Roh IK, Kim IJ, Chung JH and Byun SM: Affinity purification and binding characteristics of Citrobacter freundii AmpR, the transcriptional regulator of the ampC beta-lactamase gene. Biotechnol Appl Biochem 23: 149-154, 1996.

12. Jacobs C, Huang LJ, Bartowsky E, Normark S and Park JT: Bacterial cell wall recycling provides cytosolic muropeptides as effectors for beta-lactamase induction. EMBO J 13: 4684-4694, 1994.

13. Li JB, Cheng J, Yin J, et al: Progress on AmpC beta-lactamases. Curr Bioinform 4: 218-225, 2009.

14. Jacoby GA: AmpC beta-lactamases. Clin Microbiol Rev 22: $161-182,2009$

15. Schmidtke AJ and Hanson ND: Model system to evaluate the effect of ampD mutations on AmpC-mediated beta-lactam resistance. Antimicrob Agents Chemother 50: 2030-2037, 2006.

16. Khanal S, Joshi DR, Bhatta DR, Devkota U and Pokhrel BM: $\beta$-lactamase-producing multidrug-resistant bacterial pathogens from tracheal aspirates of intensive care unit patients at National Institute of Neurological and Allied Sciences, Nepal. ISRN Microbiol 2013: 847569, 2013.

17. Pai H, Kang CI, Byeon JH, et al: Epidemiology and cahnical features of blood stream infections caused by AmpC-type-betalactamase-producing Klebsiella pneumoniae. Antimiciob Agents Chemother 48: 3720-3728, 2004

18. Moland ES: Newer $\beta$-Lactamases: clinical and laboratory implications, Part II. Clinical Microbiology Newsletter 30: 79-85, 2008

19. Moland ES: Newer $\beta$-Lactamases: clinical and laboratory implications, Part I. Clinical Microbiology Newsletter 30: 71-77, 2008

20. GniadkowskiM:Evolutionandepidemiology of extended-spectrum beta-lactamases (ESBLs) and ESBL-producing microorganisms. Clin Microbiol Infect 7: 597-608, 2001.

21. Lodge J, Busby S and Piddock L: Investigation of the Pseudomonas aeruginosa ampR gene and its role at the chromosomal ampC beta-lactamase promoter. FEMS Microbiol Lett 111: 315-320, 1993.

22. Campbell JI, Ciofu O and Høiby N: Pseudomonas aeruginosa isolates from patients with cystic fibrosis have different beta-lactamase expression phenotypes but are homogeneous in the ampC-ampR genetic region. Antimicrob Agents Chemother 41: 1380-1384, 1997

23. Poirel L, Guibert M, Girlich D, Naas T and Nordmann P: Cloning, sequence analyses, expression, and distribution of ampC-ampR from Morganella morganii clinical isolates. Antimicrob Agents Chemother 43: 769-776, 1999.
24. Balasubramanian D, Schneper L, Merighi M, et al: The regulatory repertoire of Pseudomonas aeruginosa AmpC B-lactamase regulator AmpR includes virulence genes. PLoS One 7: e34067, 2012.

25. Balcewich MD, Reeve TM, Orlikow EA, et al: Crystal structure of the AmpR effector binding domain provides insight into the molecular regulation of inducible ampc beta-lactamase. J Mol Biol 400: 998-1010, 2010.

26. Nakano R, Okamoto R, Nakano Y, et al: CFE-1, a novel plasmid-encoded AmpC beta-lactamase with an ampR gene originating from Citrobacter freundii. Antimicrob Agents Chemother 48: 1151-1158, 2004.

27. Lee Y, Choi H, Yum JH, et al: Molecular mechanisms of carbapenem resistance in Enterobacter cloacae clinical isolates from Korea and clinical outcome. Ann Clin Lab Sci 42: 281-286, 2012.

28. Fortineau N, Poirel L and Nordmann P: Plasmid-mediated and inducible cephalosporinase DHA-2 from Klebsiella pneumoniae. J Antimicrob Chemother 47: 207-210, 2001.

29. Decré D, Verdet C, Raskine L, et al: Characterization of CMY-type beta-lactamases in clinical strains of Proteus mirabilis and Klebsiella pneumoniae isolated in four hospitals in the Paris area. J Antimicrob Chemother 50: 681-688, 2002.

30. Carrasco-López C, Rojas-Altuve A, Zhang W, et al: Crystal structures of bacterial peptidoglycan amidase AmpD and an unprecedented activation mechanism. J Biol Chem 286: 31714-31722, 2011.

31. Lee M, Zhang W, Hesek D, et al: Bacterial AmpD at the crossroads of peptidoglycan recycling and manifestation of antibiotic resistance. J Am Chem Soc 131: 8742-8743, 2009.

32. Bauvois B: Transmembrane proteases in focus: diversity and redundancy? J Leukoc Biol 70: 11-17, 2001.

33. Lindquist S, Galleni M, Lindberg F and Normark S: Signalling proteins in enterobacterial AmpC $\beta$-lactamase regulation. Mol Microbiol 3: 1091-1102, 1989.

34. Honoré N, Nicolas MH and Cole ST: Regulation of enterobacterial cephalosporinase production: the role of a membrane-bound sensory transducer. Mol Microbiol 3: 1121-1130, 1989

35. Zhang Y, Bao Q, Gagnon LA, et al: ampG gene of Pseudomonas aeruginosa and its role in $\beta$-Lactamase expression. Antimicrob Agents Chemother 54: 4772-4779, 2010.

36. Chahboune A, Decaffmeyer M, Brasseur R and Joris B: Membrane topology of the Escherichia coli AmpG permease required for recycling of cell wall anhydromuropeptides and AmpC beta-lactamase induction. Antimicrob Agents Chemother 49: 1145-1149, 2005.

37. Schmidt H, Korfmann G, Barth H and Martin HH: The signal transducer encoded by ampG is essential for induction of chromosomal AmpC $\beta$-lactamase in Escherichia coli by beta-lactam antibiotics and 'unspecific' inducers. Microbiology 141: 1085-1092, 1995.

38. Korfmann G and Sanders CC: ampG is essential for high level expression of AmpC $\beta$-lactamase in Enterobacter cloacae. Antimicrob Agents Chemother 33: 1946-1951, 1989. 